\title{
CLXXI. THE SEPARATION OF FATTY ACIDS.
}

\author{
By ARNOLD HERBERT LEWIS. \\ From the Department of Agricultural Chemistry, University of Reading.
}

(Received October 13th, 1926.)

INTRODUCTION.

IN some preliminary experiments carried out by Professor H. A. D. Neville, the results of which had been communicated to the author, it had been noted that phenylhydrazine and hydroxylamine reacted, under suitable conditions, with the natural fats, giving crystalline products and that the yield of these products appeared to differ considerably with variation in the nature of the fat used. This gave rise to the idea that possibly one kind of glyceride, e.g. those containing saturated acids, formed solid crystalline products with the reagents mentioned above, whilst other glycerides either failed to react or gave products which were liquid or, where a solvent was used, soluble under the conditions of the experiment. The preliminary experiments had, however, been entirely qualitative and there was no information as to the degree of separation, if any, which could be obtained.

Most of the qualitative experiments had been carried out with hydrazine and phenylhydrazine and consequently the action of these reagents was first investigated. A considerable amount of work was done, but, not only did these reagents prove useless for the purpose in view, but the author was also anticipated in publication, Van Alphen [1925] publishing a complete account of these reactions.

It is only necessary, therefore, to give a very condensed account of the investigations on these lines before dealing more fully with the reaction of hydroxylamine.

\section{EXPERIMENTAL.}

Hydrazine. Falciola [1914, 1920] had investigated the action of this reagent on the fats and had come to the conclusion that in all cases hydrazides were formed but that the unsaturated fatty acids are reduced at the same time. Van Alphen, in the paper mentioned above, repeated this work under varying conditions and confirmed the conclusions of Falciola, while the author's experiments gave further proof of the fact. The experiments were discontinued, since the reaction was obviously useless for the purpose in view if, say, oleic acid gives rise to the same hydrazide as stearic acid. In addition, the products from different acids did not appear to differ sufficiently in properties to offer any hope of a basis of separation. 
Phenylhydrazine. Many earlier workers, including Baidowsky and Slepaka [1903], have noted and used the reaction of this reagent on esters in general. The reaction is mostly a simple one, though the workers mentioned above noted the formation of benzamide and other nitrogenous by-products when esters of benzoic acid were used.

In ignorance of work being carried out by Van Alphen, the author investigated the reaction of phenylhydrazine on esters of acetic, butyric, palmitic, stearic and oleic acids, both simple esters and triglycerides being used. The calculated quantities of the reagents. were heated together in an oil-bath to $150^{\circ}$ for $1 \frac{1}{2}$ hours, a stream of hydrogen being passed through the tube to prevent oxidation. After cooling and standing for some time, a solid product separated out in all cases. These products, at first red in colour, could be purified by crystallisation from alcohol. The yields were not good even when a single triglyceride was used and, this being the case, the reaction was obviously useless as a method for the separation of different acids. It was definitely ascertained that, under the conditions of experiment, phenylhydrazine causes no reduction of the unsaturated to saturated acids. Thus the product obtained from triolein and phenylhydrazine gave on hydrolysis a fatty acid with the iodine value of pure oleic acid.

Hydroxylamine. The two previous reagents having proved useless for the purpose in view, attention was turned to hydroxylamine. The action of this reagent on the triglycerides of the fatty acids is to give hydroxamic acids which are remarkably stable [Morelli, 1908]. In the preparation of stearohydroxamic acid from tristearin by the action of hydroxylamine in the presence of sodium ethylate, Morelli found that the sodium salt of stearohydroxamic acid separated out in practically theoretical quantity from alcohol.

The method adopted in the following work is essentially the same as that used by Morelli, only slight alterations in procedure being found of advantage.

Stearohydroxamic acid, $\mathrm{C}_{17} \mathrm{H}_{35} \mathrm{CO}$. $\mathrm{NH} . \mathrm{OH}$. Since this acid is fairly easily prepared and as its sodium salt is insoluble in alcohol, it was decided to prepare a sample of it for comparison purposes. Hydroxylamine hydrochloride, $1 \cdot 8 \mathrm{~g}$. in boiling methyl alcoholic solution, was added to $1 \cdot 3 \mathrm{~g}$. of sodium in absolute ethyl alcohol. To this solution $5 \mathrm{~g}$. of tristearin were added and the whole thoroughly shaken for some time. After an hour the whole was warmed on a water-bath to dissolve any solid which might have formed. After allowing to stand for 24 hours, an abundant white precipitate of sodium stearohydroxamate was formed. This was filtered off, washed well with alcohol and then dried over sulphuric acid. The suspension of the dry salt in water was gradually raised to boiling-point when a turbid liquid was obtained, showing that slight hydrolysis had occurred. The solution was then acidified with $N$ acetic acid with constant stirring, using Congo red as indicator. After standing for an hour the precipitate was filtered off and washed well with water. The solid was then roughly dried between filter papers and recrystallised from alcohol three times. It was finally washed with ether and dried, when the solid stearo- 
hydroxamic acid was obtained in the form of white crystals which gave a violet colour with ferric chloride in alcohol; M.P. $104^{\circ}$ (Morelli, 104 ${ }^{\circ}$ ).

Palmitohydroxamic acid, $\mathrm{C}_{15} \mathrm{H}_{31} \mathrm{CO} . \mathrm{NH}$. OH. This acid was prepared, using exactly the same amounts of reagent as for stearohydroxamic acid. A white crystalline compound was obtained which, after recrystallising from alcohol three times had M.P. $100^{\circ}$ (Morelli, $99^{\circ}$ ). It gave a violet colour with ferric chloride in alcohol. The yield of sodium palmitohydroxamate and of the palmitic acid obtained from this by hydrolysis was practically theoretical and this fact seemed to indicate that we might have a method of separating these acids from others occurring in the natural fats. It seemed probable that the hydroxamic acids from the unsaturated and lower fatty acids might either be much more soluble or might even be unstable.

Butyrohydroxamic acid, $\mathrm{C}_{3} \mathrm{H}_{7} . \mathrm{CO} . \mathrm{NH} . \mathrm{OH}$. To test whether the lower fatty acids give hydroxamic acids under the conditions described above and, if so, to test their solubility, the preparation of butyrohydroxamic acid was attempted.

Using exactly the same procedure as for stearohydroxamic acid, it was found that no solid separated even after allowing to stand for a week. Either sodium butyrohydroxamate is completely soluble in alcohol or it is not formed under the given conditions. To test this point, the sodium chloride was filtered off and the filtrate evaporated to dryness on a water-bath. On adding acetic acid to the solution in water of the solid obtained by evaporation, a vigorous evolution of nitrogen occurred. It seems probable that sodium butyrohydroxamate was formed but is easily decomposed.

It may be concluded from the above that mixtures of higher and lower saturated fatty acids should be easy of separation by this method. A trial was made with a mixture of $4 \cdot 229 \mathrm{~g}$. tristearin $(=4 \cdot 105 \mathrm{~g}$. stearic acid) and $1 \cdot 387 \mathrm{~g}$. tributyrin. A solution of $1 \cdot 8 \mathrm{~g}$. of $\mathrm{NH}_{2} \mathrm{OH} . \mathrm{HCl}$ in $25 \mathrm{cc}$. methyl alcohol was added while boiling to $1 \cdot 3 \mathrm{~g}$. sodium in $45 \mathrm{cc}$. ethyl alcohol. To this the above weights of triglycerides were added and the whole well shaken. After an hour the mixture was warmed on a water-bath to dissolve any solid which had separated out. It was then allowed to stand for 24 hours. The solid separating was filtered off and washed with 5 cc. alcohol. This solid was dried over sulphuric acid and finally hydrolysed by dilute mineral acid. The fatty acid so obtained was solid. It was extracted with ether and, when dried, weighed $4.035 \mathrm{~g}$. The theoretical yield of stearic acid is $4.105 \mathrm{~g}$. Thus a practically quantitative separation of stearic and butyric acids was effected.

Since butyric acid can be easily separated from stearic acid by the above method, it was decided to repeat the experiment with an acid of the same series intermediate between butyric and palmitic acids.

Caprylohydroxamic acid, $\mathrm{C}_{7} \mathrm{H}_{15} \mathrm{CO} . \mathrm{NH}$. OH. It was not possible to obtain the triglyceride of caprylic acid in a pure state, and the ethyl ester was used.

Adopting the usual procedure, it was found that no solid matter (sodium caprylohydroxamate) separated out on standing, and it was concluded that 
sodium caprylohydroxamate is soluble in alcohol. It should, therefore, be possible to separate stearic and caprylic acids by this method. To test the point a mixture of $1.917 \mathrm{~g}$. tristearin $(1 \mathrm{~mol}$.) and $1.111 \mathrm{~g}$. ethyl caprylate ( 3 mols.) was added to the solution of $\mathrm{NH}_{2} \mathrm{OH}$ and sodium ethylate prepared as before. After standing for 24 hours the solid was filtered off, dried over sulphuric acid and then hydrolysed by dilute mineral acid. The fatty acid so obtained was extracted with ether and dried.

This acid weighed $1.825 \mathrm{~g}$. whereas the theoretical yield is $1.835 \mathrm{~g}$. stearic acid. The filtrate obtained above was evaporated to dryness on a water-bath and then hydrolysed by dilute mineral acid, extracted with ether and the solution dried. On distillation, a yield of $0.9055 \mathrm{~g}$. of caprylic acid was obtained. The theoretical yield is $0.930 \mathrm{~g}$. The separation of caprylic and stearic acids appears then to be quantitative. The fact that the yield of caprylic acid is not quite quantitative is probably due to the fact that this acid is somewhat soluble in water and the ether extraction was not complete.

Laurohydroxamic acid, $\mathrm{C}_{11} \mathrm{H}_{23} \mathrm{CO} . \mathrm{NH} . \mathrm{OH}$. Since a separation of caprylic and stearic acids was satisfactory, it was decided to repeat the experiment with an acid still higher in the same series. For this purpose lauric acid was chosen.

As with caprylic acid, it was impossible to obtain the triglyceride in a pure state so the ethyl ester was used. In the first case, an attempt was made to prepare laurohydroxamic acid using the same method as for stearohydroxamic acid. A white crystalline substance was obtained which after repeated recrystallisation from alcohol had M.P. $82 \cdot 5^{\circ}$. It gave a violet colour with ferric chloride in alcohol, was soluble in warm alcohol, fairly soluble in ether and practically insoluble in dilute alcohol. Analysis established that the substance was pure laurohydroxamic acid which from an examination of the literature does not appear to have been prepared before. The yield, however, was low and it was obvious that a theoretical separation of stearic and lauric acids by this method was impossible. The approximate limit of application of this method for the separation of the higher from the lower saturated fatty acids is plain. It lies somewhere between the $\mathrm{C}_{8}$ and the $\mathrm{C}_{12}$ acids.

Oleohydroxamic acid, $\mathrm{C}_{17} \mathrm{H}_{33} \mathrm{CO} . \mathrm{NH} . \mathrm{OH}$. An investigation of the action of hydroxylamine on oleic acid was next undertaken in the hope that oleohydroxamic acid would differ sufficiently from the corresponding stearic acid compound to enable a separation to be effected.

A methyl alcohol solution of $1.8 \mathrm{~g}$. $\mathrm{NH}_{2} \mathrm{OH}$. $\mathrm{HCl}$ was added whilst boiling to $1.3 \mathrm{~g}$. sodium in $45 \mathrm{cc}$. ethyl alcohol. To this solution $5 \mathrm{~g}$. triolein were added and the whole was well shaken. After one hour's standing it was warmed on a water-bath. Even after allowing to stand for several days no separation of solid matter took place. This might have been due to the fact that sodium oleohydroxamate is soluble in alcohol or it might not be formed under the conditions used.

Repeating the experiment with $10 \mathrm{cc}$. methyl alcohol and $25 \mathrm{cc}$. ethyl 
alcohol and the same weights of reagents and triolein, a small yield of sodium oleohydroxamate was obtained. The free hydroxamic acid is a white crystalline substance; M.P. $61^{\circ}$ (Morelli, $61^{\circ}$ ). It gives a violet colour with ferric chloride in alcohol. On hydrolysis with dilute sulphuric acid, oleic acid was obtained which had a theoretical iodine value.

From the above it is seen that hydroxylamine has no effect on the double bond of oleic acid. The action of hydroxylamine on other unsaturated acids has been studied by several workers [Rostoski, 1875; Thiele and Pickard, 1899; Posmir, 1903; Harries and Haarman, 1904]. With esters of higher acids at ordinary temperatures the evidence points to the direct formation of the hydroxamic acids, no reaction occurring between the hydroxylamine molecule and the unsaturated linkage. Oleic acid would seem to be normal in this respect.

Separation of stearic and oleic acids. Since sodium oleohydroxamate is fairly soluble in alcohol, a method of separating oleic acid from the higher saturated fatty acids based upon the action of hydroxylamine on the fatty acids should be possible, providing enough solvent is present to keep the sodium oleohydroxamate in solution.

To test this point a mixture of tristearin and triolein was used. Equimolecular amounts of these two substances were acted upon by enough hydroxylamine to combine with both. After treatment as for stearohydroxamic acid a substance was obtained which gave a violet colour with ferric chloride in alcohol and had M.P. $95^{\circ}$. On recrystallising again the melting point rose to $101^{\circ}$. The substance was then hydrolysed by dilute mineral acid and the fatty acid so obtained extracted with ether and dried. This acid was solid; M.P. $68 \cdot 5^{\circ}$ (stearic acid, M.P. $69^{\circ}$ ). It gave no iodine value.

There remained to test whether a quantitative separation of oleic and stearic acids is possible by this method. An equimolecular mixture of tristearin and triolein was treated with enough hydroxylamine to combine with all the triglycerides present. To $1.8 \mathrm{~g}$. $\mathrm{NH}_{2} \mathrm{OH} . \mathrm{HCl}$ in $25 \mathrm{cc}$. methyl alcohol were added while boiling $1.3 \mathrm{~g}$. sodium in $45 \mathrm{cc}$. ethyl alcohol. To this were added $1.522 \mathrm{~g}$. triolein $(=1.456 \mathrm{~g}$. oleic acid) and $1.532 \mathrm{~g}$. tristearin $(=1.467 \mathrm{~g}$. stearic acid). The whole was shaken thoroughly and after standing for an hour was warmed on a water-bath. After 24 hours' standing some solid matter had separated out. This was filtered off. The solid was dried over sulphuric acid and finally hydrolysed by dilute mineral acid when it gave a fatty acid which was extracted with ether and dried. The weight of this acid obtained from the solid precipitate was $1.461 \mathrm{~g}$.: theory requires $1.467 \mathrm{~g}$. stearic acid.

The filtrate obtained was evaporated to dryness on a water-bath. The oil so obtained was hydrolysed by dilute mineral acid, extracted with ether and dried.

Yield: 1.459 g. fatty acid: theory requires $1 \cdot 456 \mathrm{~g}$. oleic acid.

It would therefore appear that the method gives complete separation of stearic and oleic acids. 
For a perfect separation it is obvious that the weights of triglycerides and reagents, and the volume of solvent must be within certain limits. It was decided to keep the weights of reagents and the volume of alcohol fixed at $1.8 \mathrm{~g}$. of $\mathrm{NH}_{2} \mathrm{OH} . \mathrm{HCl}$ in $25 \mathrm{cc}$. methyl alcohol and $1.3 \mathrm{~g}$. sodium in $45 \mathrm{cc}$. ethyl alcohol. Repeating the experiment described above, but using greater amounts of triglycerides for the same volume of solvent and weights of reagent, a less perfect separation was obtained. In one experiment where $2.001 \mathrm{~g}$. triolein $(=1.915 \mathrm{~g}$. oleic acid $)$ and $2.014 \mathrm{~g}$. tristearin $(=1.928 \mathrm{~g}$. stearic acid $)$ were the amounts used, there were obtained $2 \cdot 110 \mathrm{~g}$. of solid acid, indicating that some sodium oleohydroxamate had separated with the stearic acid compound.

Separation of palmitic and oleic acids. $1 \cdot 8 \mathrm{~g} . \mathrm{NH}_{2} \mathrm{OH} . \mathrm{HCl}$ in 25 cc. methyl alcohol were added while boiling to $1.3 \mathrm{~g}$. sodium in $45 \mathrm{cc}$. ethyl alcohol. A mixture of $1.5656 \mathrm{~g}$. triolein $(=1.498 \mathrm{~g}$. oleic acid) and $1.4275 \mathrm{~g}$. tripalmitin $(=1 \cdot 360 \mathrm{~g}$. palmitic acid) was added. After 24 hours' standing the solid formed was filtered off, dried over sulphuric acid and hydrolysed by dilute mineral acid. The fatty acid so formed was extracted with ether and when dried weighed $1.337 \mathrm{~g}$. The filtrate from the above was evaporated to dryness on a water-bath, hydrolysed, etc., and gave fatty acid weighing $1.513 \mathrm{~g}$. The separation of palmitic and oleic acids appears to be as complete as in the case of stearic and oleic acids.

With smaller amounts of triglycerides than those used above the separation was less perfect. A mixture of $0.9418 \mathrm{~g}$. triolein and $0.8485 \mathrm{~g}$. tripalmitin was tried, using the same weights of reagents and volume of solvent as in the previous experiment. The solid fatty acid obtained weighed only $0.602 \mathrm{~g}$., whereas the theoretical yield would be $0 \cdot 808 \mathrm{~g}$. palmitic acid. Thus the solubility of sodium palmitohydroxamate becomes of importance when only small amounts of tripalmitin are used.

With weights of triglycerides greater than those used in the first experiment, the separation was again not perfect. In one experiment with the same weights of reagents and the same volume of solvent, a mixture of $2.7646 \mathrm{~g}$. triolein and $2 \cdot 5200 \mathrm{~g}$. tripalmitin was tried. The solid formed after 24 hours' standing was treated as in previous experiments and gave $2 \cdot 6654 \mathrm{~g}$. solid fatty acid, whereas only $2 \cdot 4020 \mathrm{~g}$. palmitic acid are theoretically required.

From the above trials it is seen that a quantitative separation of stearic and oleic acids and of palmitic and oleic acids can be accomplished by the method described, provided that the weights of substances used are adjusted to the weights of reagents and volume of solvent used. This method is quite simple and the only precaution necessary is to use only absolutely pure and dry alcohol and to keep the apparatus used fitted with good calcium chloride tubes. After the sodium salt of the hydroxamic acid has once been prepared there is no further need to exclude moisture.

Variation in the proportion of glycerides. Both in the case of the stearicoleic mixture and the palmitic-oleic mixture, experiments were tried with 
varying proportions of saturated to unsaturated acid. The proportions used varied between three parts of saturated to one of unsaturated glyceride and one part of saturated to three parts of unsaturated glyceride. The separation was satisfactory in all cases when the total concentration of the fats in the mixed alcohol solvent was kept at about 4-5\%.

Application to natural oil. The method was tried with palm oil and castor oil, an indication of the extent of the separation being obtained from the iodine value of the products. A hot solution of $1 \cdot 8 \mathrm{~g}$. $\mathrm{NH}_{2} \mathrm{OH} . \mathrm{HCl}$ in $25 \mathrm{cc}$. methyl alcohol was added to $1 \cdot 3 \mathrm{~g}$. sodium in $45 \mathrm{cc}$. ethyl alcohol. To this solution were added $4 \mathrm{~g}$. palm oil. The reaction mixture was worked up as in previous cases. The iodine values of the original oil and of the two products were then determined. A similar experiment was carried out using $4 \mathrm{~g}$. of castor oil. The results obtained may be given in tabular form:

$\begin{array}{lccc} & \begin{array}{c}\text { Iodine value } \\ \text { of oil }\end{array} & \begin{array}{c}\text { Iodine value of acid } \\ \text { from solid precipitate }\end{array} & \begin{array}{c}\text { Iodine value of } \\ \text { acid from filtrate }\end{array} \\ \text { Palm oil } & 19.53 & \text { Nil } & 26.53 \\ \text { Castor oil } & 83.39 & \text { Nil } & 100.03\end{array}$

It cannot be proved from the above that a perfect separation has occurred, as some saturated acids may have remained in solution, making the iodine value of the fatty acid obtained from the filtrate low. However, the separation is satisfactory so far as it gives saturated acids only in the precipitated hydroxamic acid.

Hydroxamic acids from hydroxy-fatty acids. As hydroxy-fatty acids quite commonly occur in nature, it was considered desirable to investigate them from the point of view of this reaction, i.e. to see if they gave sodium hydroxamates which were either soluble or insoluble in alcohol. It was decided to use a natural oil having a fairly high acetyl value for this purpose and rape oil was chosen. A sample of oil was first saponified by alcoholic potash and the free fatty acids obtained. After drying, the acetyl value of these fatty acids was determined and found to be $16 \cdot 1$.

The oil was then acted upon by hydroxylamine in the presence of sodium ethylate as in the experiments previously described, $5 \cdot 3067 \mathrm{~g}$. rape oil being used. The solid precipitate obtained after 24 hours' standing gave $0.5217 \mathrm{~g}$. fatty acid on hydrolysis. This fatty acid had no acetyl value. The filtrate from the above, after working up in the usual way, gave fatty acids of acetyl value 18.3. It would appear therefore that the hydroxy-acids present in rape oil give hydroxamic acids whose sodium salts are soluble in alcohol and the above method can be used for separating hydroxy-fatty acids from acids which give hydroxamic acids having insoluble sodium salts.

\section{Summary.}

1. Phenylhydrazine reacts with the fatty acids and their triglycerides to give the corresponding phenylhydrazides. This applies to both saturated and unsaturated acids, the double bonds of the latter not being attacked, but the 
products, owing to the great similarity of their solubility in various media, are unsuitable as a means of separating fatty acids.

2. Hydroxylamine, in the presence of sodium ethylate, reacts with the triglycerides of the fatty acids to give stable hydroxamic acids.

3. The sodium salts of the hydroxamic acids from palmitic and stearic acids are completely insoluble in alcohol under the conditions stated but, with decrease of molecular weight, the solubility increases, the corresponding compound from lauric acid being appreciably soluble whilst that from caprylic acid is totally soluble.

4. The sodium salts of the hydroxamic acids from the unsaturated fatty acids and from the hydroxy-fatty acids are completely soluble under the given conditions.

5. The reaction, therefore, offers a method of separation of saturated fatty acids of high molecular weight, certainly from palmitic acid upwards from (a) the lower acids such as butyric and caprylic, $(b)$ the hydroxy-acids, and (c) the unsaturated acids, in cases where all these may occur in natural oils in the form of glycerides.

6. For the separation to be satisfactory, attention must be paid to the concentration of the reagents, a concentration of about $4-5 \%$ in the mixed alcohols being the best. In addition, the methyl and ethyl alcohol used, particularly the former, must be pure and dry.

The author is indebted to the Research Board of the University for a grant in aid of this work and to Professor H. A. D. Neville for his interest in it throughout.

\section{REFERENCES.}

Van Alphen (1925). Rec. Trav. Chim. 44, 1064.

Baidowsky and Slepaka (1903). J. Russ. Phys. Chem. Soc. 35, 68.

Falciola (1914). Ann. Chim. Applic. 2, 351.

- (1920). Gazz. Chim. Ital. 50, 162.

Harries and Haarman (1904). Ber. deutsch. chem. Ges. 37, 252.

Morelli (1908). Atti R. Acad. Lincei, 17, ii, 74.

Posmir (1903). Ber. deutsch. chem. Ges. 36, 4305.

Rostoski (1875). Liebig's Annalen, 178, 213.

Thiele and Pickard (1899). Liebig's Annalen, 309, 189. 